\title{
Nanotechnology versus coronavirus
}

\author{
In the face of the coronavirus pandemic, it is time for the nanotechnology community to shine and build on its \\ experience with nanoscale materials and drug delivery to provide knowledge and tools for COVID-19 vaccine and \\ therapeutics development.
}

W hile many parts of the world remain in lockdown and universities are closed, numerous nanotechnology research labs have kept open throughout the last months to redirect their efforts towards the development of a vaccine and/or nanoformulations of therapeutics for the treatment of COVID-19. Expertise in nucleic acid and drug delivery, cancer nanovaccines, immunoengineering, nanotechnology-based sensors and platform technologies positions nanotechnology in a unique place to tackle some of the key issues in pre-clinical and clinical COVID-19 research. After all, viruses are naturally occurring nanoparticles, and indeed, the nanotechnology community has long been trying to capitalize on the properties of viruses and mimic their behaviour, for example, for the design of virus-like nanoparticles for targeted drug delivery and gene editing.

In this Focus issue, nanotechnologists from different areas discuss ongoing efforts in COVID-19 vaccine development, the potential advantages of nanoformulations for the most promising therapeutic candidates, nanotechnological tools that may facilitate worldwide vaccine manufacturing and distribution, and nanomaterial-based viral disinfectants and detection platforms.

Vaccines are the most promising solution to mitigate the spread of the coronavirus SARS-CoV-2. Nanomaterials play an important role in all aspects of vaccine design, delivery and administration. Nanoparticles enable multivalent antigen presentation and stabilization of antigens upon administration, they can serve as adjuvants to boost the immune response, and they can act as carriers for the targeted delivery of antigens. Indeed, an mRNA vaccine delivered by a liposomal nanoparticle is amongst the candidates currently in clinical trials against SARS-CoV-2. While it remains a fact that no mRNA or DNA vaccine is currently approved for any disease, the delivery of nucleic acids requires some form of modification or a nanodevice to prevent degradation in the body, and liposomal devices have indeed already been approved for RNA delivery, albeit not yet for vaccines. The nanotechnology community has further gained immense knowledge over the past years in the development of cancer nanovaccines to boost and/or reprogram host immunity, which may now provide a solid starting point for immune-mediated approaches against SARS-CoV-2.

Importantly, nanomaterial-based platform technologies may also play a key role in the distribution and administration of vaccines in resource-poor or densely populated developing countries by enabling self-administration, for example, through microneedle patches, single-dose slow-release implants, film-based vaccines, or by using plant viral nanoparticles for antigen delivery, which do not require cold chains. In a global pandemic, in which healthcare systems are operating at the limit, such self-administration technologies may prove extremely valuable. Cold chain requirements can also be circumvented by applying alternative production platforms, such as molecular farming in plants. Thereby, vaccines are produced in edible leaf tissue for the vaccination of humans and livestock.

From a therapeutic standpoint, the repurposing of existing drugs is currently at the centre of research owing to a lack of new therapeutic treatments against COVID-19. So far, only a few drugs have demonstrated efficacy against COVID-19 in clinical trials. The anti-inflammatory corticosteroid dexamethasone has recently been shown to have life-saving efficacy in a clinical trial. Dexamethasone has previously been formulated as a nanomedicine for the treatment of other diseases; for example, liposomal dexamethasone has shown efficacy in multiple myeloma. In the case of COVID-19, a nanomedicine formulation may enable the targeting of alveolar macrophages upon pulmonary delivery or of phagocytes at inflammation sites following intravenous administration. Moreover, the anti-fibrotic effects of dexamethasone can be potentiated by reformulating it as a nanomedicine. The argument can be made, of course, that a nanoformulation will make the therapy even more expensive (not even speaking of the need for additional clinical trials); however, if such nanoformulation improves clinical outcomes, and thus, would allow critically ill patients to leave the intensive care unit earlier, the cost reduction in hospitalization and faster patient recovery may be well worth it.

A main reason for the ongoing repurposing of drugs and fast-tracking of preclinical and clinical testing of vaccine formulations for COVID-19, is the lack of time. The development, clinical testing and approval of new drugs usually take years. Therefore, in the future, it will be important to scientifically and financially invest in platform technologies that can then be rapidly repurposed on demand, to be better prepared for future outbreaks - a lesson (hopefully) learned from the current pandemic. Nanodevices and nanoformulations offer such platform technologies, and a thorough investigation of their fundamental science and biological interactions will be of great value for the future development of vaccines and therapeutics.

Facing the SARS-CoV-2 pandemic, the nanotechnology community has joined forces and provides their tools and experience to the COVID-19 research efforts. There has been criticism about the clinical impact of nanotechnology, but the time has now come to highlight the knowledge and previous experience of nanotechnologists in vaccine and drug development, delivery and distribution. The ongoing research efforts and collaborations with the clinic and industry will hopefully have an impact on the future of our field to further open the way for the clinical translation of nanotechnology. However, while lockdowns are starting to be eased, let us not forget that a vaccine may still be far away from clinical reality and that the road from preclinical testing to clinical efficacy can be long and bumpy - a fact that the nanotechnology community is well aware of.

Published online: 6 August 2020 https://doi.org/10.1038/s41565-020-0757-7 